\title{
Eine Ionenquelle mittels Hochfrequenzentladung ${ }^{1}$
}

\author{
Von Hugo Neuert * \\ (Z. Naturforschg. 4a, 449-455 [1949]; eingegangen am 6. Oktober 1948)
}

\begin{abstract}
Es wird eine Ionenquelle beschrieben, bei der die Ionen durch eine elektrodenlose Hochfrequenzgasentladung bei Drucken von $1-10 \cdot 10-3 \mathrm{~mm}$ erzeugt und durch eine Gleichspannung bis zu $10 \mathrm{kV}$ aus dem Entladungsgefäß herausgezogen werden. Mit einer solchen Entladung lassen sich leicht Ionenströme von etwa $15 \mathrm{~mA}$ erzielen. Die erreichbaren Stromstärken können bei gleichbleibender Senderleistung von 50-100 W noch beträchtlich erhöht werden durch Verwendung eines schwachen, praktisch die ganze als Entladungsgefăls dienende Kugel durchsetzenden Magnetfeldes. Dabei kann der Druck im Entladungsgefäß auf weniger als die Hälfte des Druckes bei Betrieb ohne Magnetfeld gesenkt werden. Es wird gezeigt, daß die Gasentladung dem Sender nur wenige Prozent der Senderverlustleistung entzieht. Verwendet man statt der kontinuierlichen Spannung einen vorher auf $10-20 \mathrm{kV}$ aufgeladenen Kondensator, so erhält man durch die Kondensatorentladung Stromstöße von $1-2 \cdot 10^{-3}$ Coulomb während einer Entladungsdauer von etwa $10^{-2}$ sec.

Infolge des verhältnismäßig niedrigen Gasdruckes kann man den Ionenstrahl durch einen relativ weiten Kanal in einen Nachbeschleunigungsraum austreten lassen. Hinter dem Kanal wurden ohne Magnetfeld Gesamtionenströme von etwa $2 \mathrm{~mA}$, und mit Magnetfeld bis zu $3 \mathrm{~mA}$ gemessen. Es ist anzunehmen, daß diese Ströme durch Verbesserung der Ionenoptik der Anordnung noch vergrößert werden können. Die Energieverteilung und die Zusammensetzung des Ionenstrahls hinter dem Kanal (Betrieb mit Wasserstoff) ist durch elektrische und magnetische Ablenkung untersucht worden. Die elektrische Ablenkung zeigte, daß die Íonen überwiegend die gleiche Energie besitzen; diese ist bestimmt durch die Höhe der Gleichspannung. Die magnetische Ablenkung hat außerdem noch gezeigt, daß bei der hier verwendeten Versuchsanordnung etwa $20 \%$ Atomionen entstehen.
\end{abstract}

Die Ionenquelle arbeitet auch bei Dauerbetrieb stabil und zuverlässig.

$\mathrm{A}^{\prime \prime}$ $\mathrm{n}$ eine leistungsfähige Ionenquelle stellt man folgende Anforderungen:

1. Große Stromstärke hinter einem, den Entladungsraum vom Nachbeschleunigungsraum abschließenden Kanal.

2. Möglichst gleichmäßige Energie der die Ionenquelle verlassenden Ionen.

3. Einen großen Anteil an Atomionen.

4. Einen möglichst geringen Energieverbrauch.

5. Einen mäßigen Gasverbrauch.

6. Betriebssicherheit im Dauerbetrieb.

Das älteste und wohl noch immer gebräuchlichste Instrument der Ionenerzeugung für Kernumwandlungsversuche ist das Kanalstrahlrohr ${ }^{2-4}$. Allerdings lassen sich manche Einwände gegen dasselbe erheben. Der Gasverbrauch ist meist beträchtlich (etwa 20 bis $50 \mathrm{~cm}^{3}$.Torr/sec). Der

* Weil a. Rh., Marktplatz 5.

1 Eine kurze Mitteilung über die Versuche ist bereits in Z. Naturforschg. 3 a, 310 [1948] erschienen.

2 Vgl. z. B. M. Oliphant u. Lord Rutherfor d, Proc. Roy. Soc. [London] 141, 259 [1933].
Energieverbrauch ist etwa $1 \mathrm{~kW}$. Besonders unerwünscht für viele Versuche ist vor allem die Inhomogenität der Ionenenergien; es treten praktisch alle Energien von 0 bis zu der angelegten Entladungsspannung auf, mit einem Intensitätsmaximum bei etwa $2 / 3$ der Entladungsspannung. Der Anteil der Atomionen beträgt $1 / 3$ bis $2 / 3$ der Gesamtionenzahl, er ist im Vergleich zu anderen Ionenquellen beträchtlich. Der erzielbare Gesamtionenstrom beträgt etwa $1,5 \mathrm{~mA}$.

Vor allem gab die Inhomogenität der Energie der Kanalstrahlen Anlaß zur Konstruktion anderer Ionenquellen. Mit Ionenquellen mittels Niedervolt- oder Kapillarbogen sind recht homogene Ionen, meist aber nur mäßige Intensitäten erzielt worden ${ }^{5}$. Neuerdings finden oft Ionenquellen Verwendung, bei denen die Ionisierung durch Elek-

3 C. H a i l e r, Veröff. Siemens-Konzern 17, 115 [1938].

\& W. B othe u. W. Gentne r, Z. Physik 104, 685 [1937].

5 Vgl. z. B. H. K o r s ching, Physik. Z. 42, 73 [1941]. 
tronenstrahlen erzwungen wird. Die Leistung einer solchen Ionenquelle hängt sehr von der Leistungsfähigkeit der die Elektronen emittierenden Kathode ab; hierdurch wird die Konstanz und die Betriebssicherheit, zumindest im Falle der kurzzeitig erreichten großen Intensität, stark beeinträchtigt ${ }^{6}$.

Im folgenden wird eine Ionenquelle beschrieben, bei der die Ionen einer elektrodenlosen Hochfrequenzentladung entstammen. Die Möglichkeit,

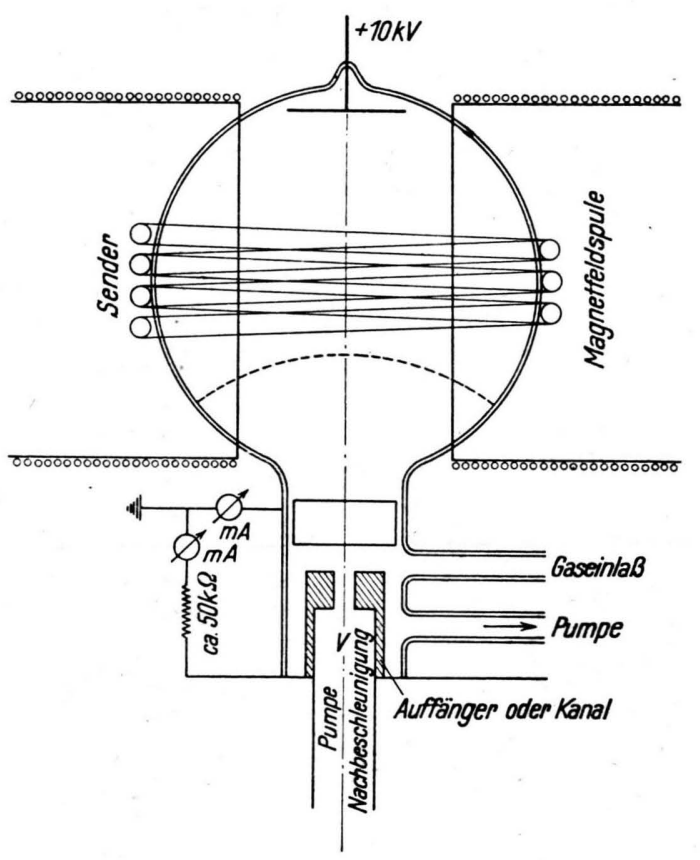

Abb. 1. Entladungsgefäß.

die Hochfrequenzentladung als Ionenquelle $\mathrm{zu}$ verwenden, wurde zuerst von $\mathrm{Th}$ on eman ${ }^{7}$ aufgezeigt. Die vorliegenden Versuche zeigen, daß eine solche Ionenquelle im Vergleich zum Kanalstrahlrohr bei durchaus ebenbürtiger Betriebssicherheit einige wesentliche Vorteile bietet.

\section{Die I o nenquelle}

Als Entladungsgefäß diente ein 2-l-Kolben aus Pyrexglas mit einem Halsansatz von $45 \mathrm{~mm}$ Weite (Abb.1). Die Entladung wurde durch eine Spule aus $\mathrm{Cu}$-Rohr von $4^{1 / 2}$ Windungen im Äquator der Kugel aufrechterhalten; die Spule war an einen Kurzwellen-

${ }^{6} \mathrm{Vgl}$ z. B. A. F inkels te in, Rev. sci. Instruments 11, 94 [1940].

7 P. C. Th o n e m a n n, Nature [London] 158, 61 [1946].

s R. W. Wo od, Physic. Rev. 35, 673 [1930]; F. K i r c hne r, Ann. Physik 77, 287 [1925]. sender angeschlossen. Die Senderleistungen betrugen bei Frequenzen von $2 \cdot 10^{7} \mathrm{~Hz}$ bis zu $150 \mathrm{~W}$.(Anodenspannungen bis zu $1000 \mathrm{~V}$ ). Die Ionen wurden durch ein elektrisches Feld von einigen $\mathrm{kV}$ aus dem Entladungsgefäß herausgezogen. Als Anode diente eine Fe-Platte. die mittels eines eingeschmolzenen W-Stiftes gegenüber dem Hals angebracht war. Die Kathode, besteherid aus einem am Eingang des Kolbenhalses angebrachten Al-Ring, war direkt geerdet. Im Kolbenhals befand sich zunächst ein $8 \mathrm{~cm}$ tiefer Auffänger für die Ionen, später der Kanal zum Austritt der Ionen in einen Nachbeschleunigungsraum. Der Auffänger war über einen Widerstand geerdet, so daß er im Betrieb eine Spannung von +500 bis 1000 Volt gegen Erde hatte.

Die Kugel wurde mit Hilfe einer 3-stufigen öldiffusionspumpe durch den Kanal ausgepumpt; durch gleichzeitige Zufuhr von $\mathrm{H}_{2}$ wurde ein (einstellbarer) Druck von $1 \cdot 10^{-3}$ bis $1,5 \cdot 10^{-2} \mathrm{~mm}$ in der Kugel aufrechterhalten. Bei einigen Versuchen wurde die Pumpleistung noch erhöht durch eine zusätzliche öldiffusionspumpe aus Glas (Sauggeschwindigkeit 4 bis $5 l /$ sec bei $10^{-3} \mathrm{~mm}$ ), die unmittelbar am Hals der Kugel angesetzt war.

\section{Die Hochfrequenzentladung}

Bei $\mathrm{H}_{2}$-Füllung trat bei Drucken von $10^{-1}$ bis $10^{-2} \mathrm{~mm}$ die bekannte, hell leuchtende Entladung auf. Die Entladungsbedingungen solcher Entladungen sind mehrfach beschrieben worden. $\mathrm{Ki}$ r h n e ${ }^{8}$ hat gezeigt, daß sich solche Entladungen auch noch bei niedrigen Drucken aufrecht erhalten lassen. Die Leuchterscheinungen nehmen bei abnehmendem Druck rasch ab. Besonders sorgfältig sind die Vorgänge des Zündens solcher Entladungen, die Lage des Zündspannungsminimums und seine Abhängigkeit vom Gasdruck und von der Wellenlänge studiert worden ${ }^{8,9}$. Nach neueren Untersuchungen sind die Gefäßdimensionen sowie die Glasart von wesentlichem Einfluß ${ }^{10}$. Die Untersuchungen haben gezeigt, daß bei Frequenzen von $1-2 \cdot 10^{7} \mathrm{~Hz}$ eine Zündung bei $\mathrm{H}_{2}$-Drucken von $10^{-2}$ bis $10^{-3} \mathrm{~mm}$ für den Fall der hier verwendeten Gefäßdimensionen und bei den zur Verfügung stehenden Feldstärken ohne weiteres gewährleistet ist. Es sind im Betrieb des Rohrs auch nie Schwierigkeiten hinsichtlich der Zündung der Entladung aufgetreten, solange der Gasdruck größer als etwa $1 \cdot 10^{-3} \mathrm{~mm}$ war.

9 G. u. H. Gu t t o n, C. R. hebd. Séances Acad. Sci. 186, 303 [1928]; C. J. B r a s efi i ld d, Physic. Rev. 35, 1073 [1930].

10 E. W. B. Gill u. A. v. Engel, Proc. Roy, Soc. [London] 192, 446 [1948]. 
Ohne zusätzliches elektrisches Feld erfüllte die Hochfrequenzentladung die ganze Kugel praktisch gleichmäßig. Bei Anlegen des zusätzlichen Feldes zog sich die Entladung allmählich vom Kolbenhals aus nach dem oberen Teil der Kugel

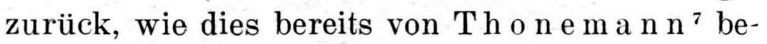
schrieben worden ist. Bei einer Spannung von $8 \mathrm{kV}$ war die Entladung etwa auf den in Abb. 1 durch eine gestrichelte Linie angegebenen Bereich zurückgegangen. Darunter befand sich ein Dunkelraum bis zum Auffänger. Auf dem Auffänger war ein schwach leuchtender Strahl, ähnlich dem Pinsel der Kanalstrahlentladungen, zu beobachten, der einen Durchmesser von einigen $\mathrm{mm}$ hatte. Bei noch niedrigeren Drucken schien der Strahl sich weiter zu verengen, er wurde gleichzeitig schwächer leuchtend.

\section{Nutzleistung der Hochfrequenz- entladung *}

Um den Einfluß der Senderleistung auf die Leistungsfähigkeit der Ionenquelle kennenzulernen, wurde gesondert gemessen, welcher Bruchteil der Senderverlustleistung von der Gasentladung aufgezehrt wird. Zu diesem Zweck wurde an die Spule ein Schwingungskreis angekoppelt, mit einem mittels Photozelle auf Leistung geeichten Glühlämpchen. Es wurde nun die Kopplung so verändert, daß im Senderkreis jeweils dieselben Strom- und Spannungswerte auftraten, die bei Betrieb der Gasentladung (bei $10 \cdot 10^{-3} \mathrm{~mm}$ ) vorhanden waren. Es zeigte sich, daß die wirklich in der Gasentladung aufgezehrte Nutzleistung linear mit der Senderverlustleistung ansteigt. Es werden dem Sender bei diesem Druck aber immer nur wenige Prozent zur Aufrechterhaltung der Gasentladung entzogen.

\section{Der Ionenstrom aus der Entlad ung}

Schon bei Drucken $>10^{-2} \mathrm{~mm}$ wurde bei Anlegen der Gleichspannung ein schwacher Strom im Auffänger festgestellt. Die Gasentladung reichte aber praktisch noch bis in den Kolbenhals hinein. Gleichzeitig wurde ein stärkerer Strom zum geerdeten Kathodenring gemessen. Bei Druckverringerung nahm der Strom zur Kathode dann

* Diese Messungen wurden zusammen mit Hrn. Dr. B. K och durchgeführt, der auch den Hochfrequenzsender zur Verfügung stellte. Für seine freundl. Mithilfe möchte ich ihm auch an dieser Stelle meinen Dank aussprechen. rasch ab, während der Strom zum Auffänger rasch anstieg. Die Entladung schnürte sich allmählich im Kolbenhals zușammen. Bei Drucken unter etwa $1 \cdot 10^{-2} \mathrm{~mm}$ war der direkt zur Kathode fließende Strom praktisch gleich Null (mit Sicherheit $<1 \mathrm{~mA}$ ). Die Beobachtung des Verschwindens des direkt zur Kathode fließenden Ionenstroms gestattete neben der visuellen Beobachtung der Entladungsverhältnisse eine Kontrolle des gew ïnschten Funktionierens der Entladung.

Bei dem vom Auffänger über den Widerstand abfließenden Strom handelte es sich zum ganz überwiegenden Teil um einen Ionenstrom. Dies wurde durch Veränderung der Tiefe des Auf-

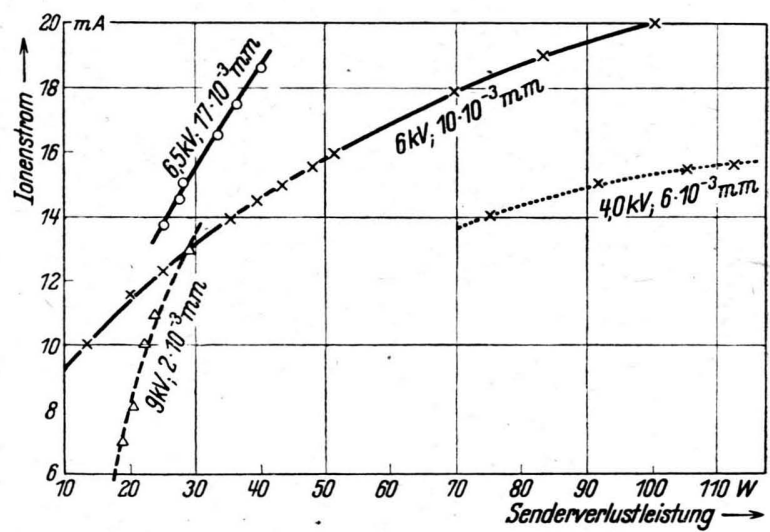

Abb. 2. Ionenstrom als Funktion der Senderleistung.

fängers sowie durch Veränderung des Ableitwiderstandes geprüft.

Mit der beschriebenen Anordnung konnte man leicht einen Ionenstrom von mehreren $\mathrm{mA}$ bei einigen $\mathrm{kV}$ Gleichspannung erhalten. Zunächst wurde die Abhängigkeit des Ionenstroms von der Senderleistung und der Höhe der Gleichspannung studiert. Der Ionenstrom nahm mit der Leistung des Senders und mit der Höhe der Spannung zu. Abb. 2 zeigt die Abhängigkeit von der Senderverlustleistung, bei konstant gehaltener Gleichspannung und möglichst konstantem Druck. Man sieht, daß der Ionenstrom stetig und zwar etwas schwächer als proportional zur Senderleistung ansteigt.

5. Der Einfluß des Gasdruckes i m Entladungsrohr

Die Entladungserscheinungen änderten sich rasch mit dem Gasdruck. Bei zu hohen Drucken, über $10 \cdot 10^{-3} \mathrm{~mm}$, war die Entladung im Kolben- 
hals noch nicht genügend eingeschnürt. Bei zu niedrigen Drucken, etwa kleiner als $1 \cdot 10^{-3} \mathrm{~mm}$, war die Entladung zu schwach, um genügend Ionen nachliefern zu können. Offensichtlich war es aber vorteilhaft, niedrige Drucke zu verwenden, weil sich dann die Einschnürung des Ionenstrahls besser ausprägte. Der günstigste Druckbereich lag bei etwa $5 \cdot 10^{-3} \mathrm{~mm}$. Im allgemeinen wird man bestrebt sein, den Gasdruck möglichst niedrig zu halten, auch im Hinblick auf die Verwendung eines Kanals gegen den Nachbeschleunigungsraum zur Aufrechterhaltung der notwendigen Druckdifferenz.

Der optimale Druckbereich hängt offenbar auch etwas von der Beschaffenheit des Glaskolbens ab.

\section{Einfluß eines zusätzlichen $\mathrm{M}$ ag netfeldes}

Um etwa am Auffänger auftretende Elektronen abzulenken, wurden gelegentlich kleine permanente Magnete angewandt. Dabei wurde beobachtet, daß bereits schwache Magnetfelder bei den verwendeten Gasdrucken eine ganz erhebliche Aufhellung der Entladung hervorrufen konnten. Es schien demnach durchaus möglich, daß die geeignete Anbringung eines Magnetfeldes eine Verbesserung der Entladungsbedingungen zur Folge haben konnte. Es wurden daher 2 Magnetspulen derart angebracht (Abb.1), daß die magnetischen Feldlinien parallel zur Ebene der Spule verliefen. Der Durchmesser der Spulen entsprach ungefähr dem Durchmesser des Kolbens. Es zeigte sich, daß bereits schwache Magnetfelder, entsprechend 20 bis 30 Ampère-Windungen/cm einen starken Effekt hervorriefen. Brannte die Entladung z. B. bei $6 \cdot 10^{-3} \mathrm{~mm}$ normal, so daß sie bei $8 \mathrm{kV}$ und $50 \mathrm{~W}$ etwa $10 \mathrm{~mA}$ Strom lieferte, so war bei einem noch recht schwachen Feld der Strom bereits auf $20 \mathrm{~mA}$ angestiegen. Bei weiterer Vergrößerung des Magnetfeldes wurde die Entladung schließlich so intensiv, daß man keinen eigentlichen Ionenstrahl mehr erhielt. Um wieder ein normales Funktionieren zu erzielen, war es notwendig, den Gasdruck herabzusetzen.

Die Verwendung eines solchen Magnetfeldes erschien also recht vorteilhaft. Dies wurde dann auch durch die Messung des Ionenstroms bestätigt. Es wurde z. B. bei einer Spannung von $8 \mathrm{kV}$ und bei einem Druck von $3 \cdot 10^{-3} \mathrm{~mm}$ mit einem schwachen Magnetfeld (entspr. 31 AmpèreWindungen/cm) eine Stromsteigerung von jeweils etwa $50 \%$ erreicht, d. h. man konnte die jeweilige Stromstärke ohne Magnetfeld nun mit Magnetfeld schon bei praktisch der halben Senderleistung erzielen.

Die Intensität der Gasentladung nahm offensichtlich mit wachsendem Magnetfeld zunächst zu. In Abb. 3 ist das Anwachsen des Ionenstroms mit der Magnetfeldstärke bei sonst konstanten Bedingungen aufgezeichnet. Optimale Arbeitsbedingungen erhielt man bei Drucken von etwa $2 \cdot 10^{-3} \mathrm{~mm}$. Bei den für den Betrieb ohne Magnetfeld günstigen Drucken von etwa $5 \cdot 10^{-3} \mathrm{~mm}$ wurde zwar bei geringem Felde noch ein vernünftiges Anwachsen

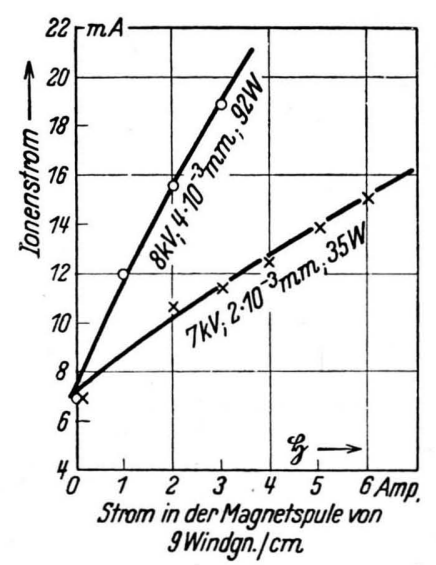

Abb. 3. Anwachsen des Ionenstroms mit der Magnetfeldstärke.

des Ionenstroms beobachtet. Bei etwas größerer Feldstärke stieg der Strom sehr rasch an, und die Entladung 'lieferte keinen gebündelten Ionenstrahl mehr.

\section{Kurzzeitige Entladungen}

Bei kontinuierlicher Hochfrequenzentladung wurde nun statt der dauernd angelegten Gleichspannung ein vorher aufgeladener Kondensator verwendet. Diese Methode hatte schon für den Fall des normalen Kanalstrahlrohrs gute Ergebnisse gebracht ${ }^{11}$. Es war dadurch möglich geworden, Ionenstöße von etwa ${ }^{1 / 100}$ sec Dauer hervorzurufen mit Stromstärken, die im Mittel etwa 10 -fach höher waren als die im Dauerbetrieb erzielten.

Die hier verwendeten Kondensatoren hatten Kapazitäten von 0,1 bis $0,4 \mu \mathrm{F}$. Sie wurden auf

11 F. Kirchner, Naturwiss. 21, 473 [1933] ; H. Neuert, Reichsber. Physik, Heft 2 [1944]. 
10 bis $20 \mathrm{kV}$ aufgeladen und in der üblichen Weise mittels einer Spaltfunkenstrecke über einen Widerstand von einigen $100 \mathrm{Ohm}$ durch die Gasentladung entladen. Der durch die Entladung entstehende Ionenstoß wurde ballistisch gemessen. Gleichzeitig wurde der am Ableitwiderstand des Auffängers

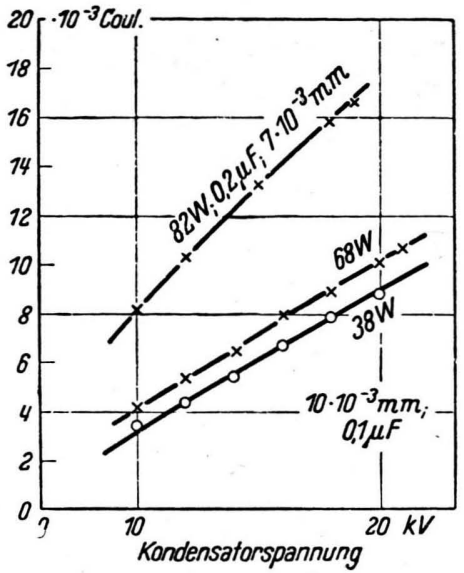

Abb. 4. Anwachsen des Stromstoßes mit der Kondensatorspannung.
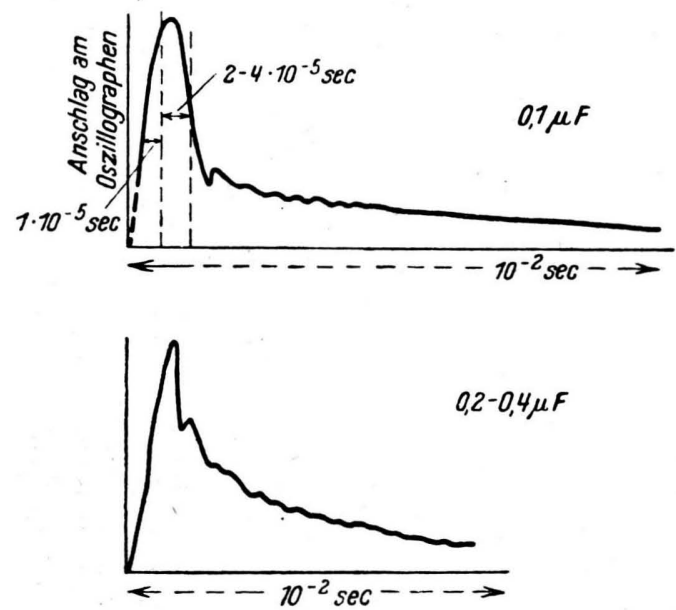

Abb. 5. Verlauf des Stromstoßes, schematisch.

entstehende Spannungsstoß auf einem Oszillographen beobachtet. Die Größe des Vorwiderstandes war nicht von wesentlichem Einfluß. Abb. 4 zeigt den Anstieg der Größe der Ionenstöße mit der angelegten Spannung im Bereiche bis $20 \mathrm{kV}$. Die Größe der Stöße steigt demnach in dem untersuchten Bereich etwa proportional zur Spannung an. Ebenso wächst die Stoßgröße proportional zur Größe der Kapazitäten an, wenigstens für den hier untersuchten Bereich bis $0,4 \mu \mathrm{F}$. Bei $0,5 \mu \mathrm{F}$ und größeren Kapazitäten bildete sich bei Spannungen über $12 \mathrm{kV}$ zwar eine starke Entladung, aber kein gebündelter Ionenstrahl mehr aus. Die Beobachtung des Stoßverlaufs auf dem Oszillographen ergab folgendes Bild: Die Stromstärke steigt in etwa $10^{-5}$ sec auf eine maximale Stromstärke an. Diese bleibt während $2-4 \cdot 10^{-5} \mathrm{sec}$ Dauer annähernd erhalten, dann sinkt die Stromstärke bei den kleinen Kapazitäten in etwa $10^{-5} \mathrm{sec}$ auf ungefähr $1 / 3$ der maximalen Stromstärke ab, der weitere Abfall erfolgt dann exponentiell, z.Tl. mit einer überlagerten schwachen Welligkeit. Abb. 5 zeigt den Stromverlauf schematisch. Die Gesamtdauer des Entladungsstoßes betrug im Mittel ${ }^{1 / 100}$ sec. Vergrößerung der Kapazität verwischte den Unterschied zwischen der Stromspitze und dem langsameren exponentiellen Abklingen allmählich; die Gesamtstoßdauer wuchs auf $1 / 50$ bis $1 / 30$ sec an.

Die Entladungsdauer und die Stoßform hingen auch vom Gasdruck ab. Bei höheren Gasdrucken schien die Entladungsdauer größer zu sein. Dafür trat die Stromspitze etwas zurück. Aus der Höhe des am Oszillographen beobachteten Spannungsstoßes kann man schließen, daß die maximale Stromstärke 200 bis $300 \mathrm{~mA}$ betrug, also das 10-15-fache der im normalen Betrieb erzielten Stromstärke.

Für die stärksten Stöße mit Kapazitäten von $0,4 \mu \mathrm{F}$ wurden bei $18 \mathrm{kV}$ während ${ }^{1 /}{ }_{50} \mathrm{sec}$ durchschnittlich Stromstärken von $150 \mathrm{~mA}$, mit Spitzenstromstärken von $250 \mathrm{~mA}$ während $4 \cdot 10^{-5} \mathrm{sec}$ erzielt.

\section{Die I o n enströme hinter einem Kanal}

In den allermeisten Fällen soll der von einer Ionenquelle gelieferte Ionenstrom noch nachbeschleunigt werden. Dazu ist es notwendig, den Entladungsraum abzuschließen; die Ionen treten dann durch einen Kanal in den Nachbeschleunigungsraum ein. Die Weite des Kanals richtet sich danach, welche Druckdifferenz aufrechterhalten werden muß. Es ist nun wichtig zu wissen, welche Ströme hinter dem Kanal noch erzielt werden können. Infolge des verhältnismäßig niedrigen Druckes im Entladungsrohr konnte ein weiter Kanal (4 mm $\varnothing, 10 \mathrm{~mm}$ lang) verwendet werden. Der austretende Strahl wurde noch um einige $\mathrm{kV}$ nachbeschleunigt und trat dann in einigen $\mathrm{cm} \mathrm{Ab-}$ stand in einen $12 \mathrm{~cm}$ tiefen Auffänger ein. Der Auf- 
fänger hatte eine Vorspannung von etwa $100 \mathrm{~V}$. Bei den Messungen der Ionenströme machte sich mitunter eine aus dem Entladungsrohr stammende Röntgenstrahlung störend bemerkbar. Sie konnte mit Zählrohren noch hinter mehr als $60 \mu \mathrm{Al}$ nachgewiesen werden. Die von dieser Strahlung ausgelösten Elektronen konnten daher z. Tl. den Auffänger trotz der angelegten positiven Vorspannung wieder verlassen und einen zu großen Ionenstrom vortäuschen. Offensichtlich trat der Ionenstrahl noch reichlich divergent aus dem Kanal aus. Die Fokussierungswirkung der Nachbeschleu-

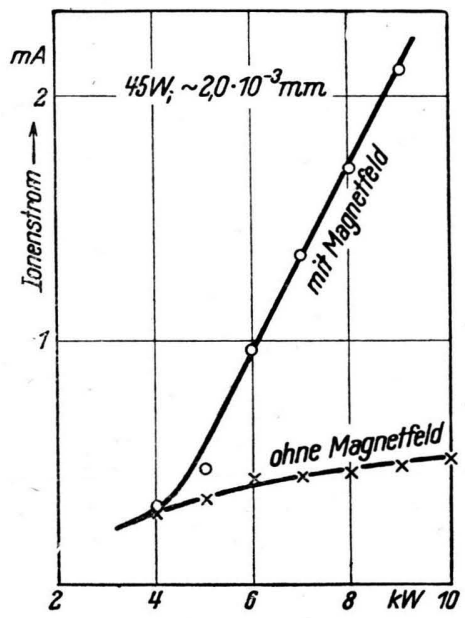

Abb. 6. Abhängigkeit des Gesamtionenstromes hinter dem Kanal von der Gleichspannung, ohne und mit Magnetfeld. Kanal $=4 \mathrm{~mm} \varnothing, 10 \mathrm{~mm}$ Länge. Abszisse: Gleichspannung.

nigungsanordnung war noch nicht optimal. Vermutlich könnten mit besserer Fokussierung um mindestens 50\% höhere Ströme gemessen werden. Die mit der genannten Anordnung und zunächst ohne zusätzliches Magnetfeld erreichten Gesamtionenströme betrugen etwa $1-2 \mathrm{~mA}$; sie stiegen mit der. Senderleistung und mit der angelegten Gleichspannung an.

Es wurde nun untersucht, ob die oben beschriebene Verbesserung der Entladungsbedingungen durch die Verwendung eines Magnetfeldes sich auch, wie zu erwarten war, auf den Gesamtionenstrom hinter dem Kanal auswirkt. Abb. 6 zeigt den Fall der Ionenströme hinter dem Kanal mit und ohne Magnetfeld, allerdings in einem gerade für die Anwendung des Magnetfeldes optimalen, d. h. ohne Magnetfeld ungünstigen Druckbereich von etwa $2 \cdot 10^{-3} \mathrm{~mm}$. Die Verbesserung durch das Magnetfeld ist hier ganz offensichtlich.
Während vorher $2 \mathrm{~mA}$ nur bei $11,5 \mathrm{kV}$ und $100 \mathrm{~W}$ erzielt wurden, genügen nun $45 \mathrm{~W}$ und $9 \mathrm{kV}$. Bei optimalen Bedingungen und bei höheren Senderleistungen konnten mit diesem Kanal Ionenströme bis zu $3 \mathrm{~mA}$ erzielt werden.

9. Untersuchung der Energieverteilung der Ionen und des Anteils an A tom-und Molekülionen

a) Elektrische Ablenkung. Um einen Überblick über die Energieverteilung der Ionen zu bekommen, wurden die Ionen zunächst un-

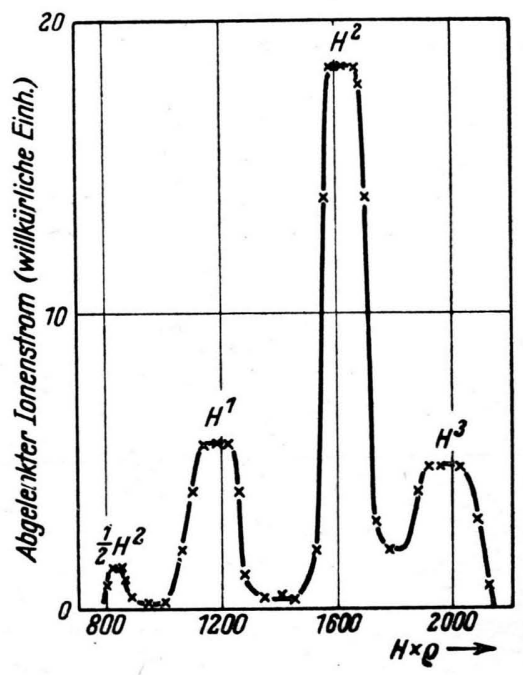

Abb. 7. Magnetisch abgelenkter Ionenstrom. Gleichspannung $5,5 \mathrm{kV}$, mit statischem Magnetfeld.

mittelbar hinter einem engen Kanal $(1,6 \mathrm{~mm} \varnothing$ und $10 \mathrm{~mm}$ Länge) durch einen Kondensator von $3 \mathrm{~cm}$ Länge und 1,1 cm Plattenabstand elektrisch abgelenkt und unter einem festen Winkel mit einem Faraday-Zylinder aufgefangen.

Die dabei gefundenen Maxima entsprachen jeweils recht gut einer Energie, die gerade durch die angelegte Gleichspannung gegeben ist. Dieser experimentelle Befund weist darauf hin, daß die Ionen im leuchtenden Teil der Entladung im oberen Bereich der Kugel entstehen. Im leuchtenden Teil der Entladung ist der Spannungsabfall der Gleichspannung nur gering. Bereits Woods hat festgestellt, daß der leuchtende Gasteil sich wie eine positive Ladungswolke verhält. Das beschleunigende Feld liegt dann praktisch zwischen der unteren Grenze der Entladung und der Kathode. Diese hier auftretende Spannung bestimmt die Ionenenergie. Man beobachtet aller- 
dings, daß die Abgrenzung der Ladungswolke mit abnehmendem Druck immer weniger ausgeprägt wird, da nun allmählich die freie Weglänge der Gasteilchen gleich oder größer wird als die Gefäßdimensionen. Umfangreiche Messungen besonders der magnetischen Ablenkung (vgl. den folgenden Abschnitt) haben aber gezeigt, daß dadurch die Homogenität der Ionenenergien nicht merklich beeinträchtigt wird.

b) Magnetische Ablenkung. Um die Zusammensetzung des Ionenstrahls kennenzulernen, wurde derselbe nach dem Austritt aus dem Kanal (ohne weitere Nachbeschleunigung) in einem sektorförmigen Magnetfeld um $60^{\circ}$ abgelenkt. Abb. 7 zeigt den abgelenkten Strom als Funktion des ablenkenden Magnetfeldes. Es zeigte sich, daß bei normalem Betrieb der Ionenquelle der Anteil der Protonen am Gesamtionenstrahl etwa $20 \%$ beträgt. Dieser Anteil schien nicht wesentlich von der Höhe der Gleichspannung abzuhängen. Dagegen scheint der Anteil der Protonen bei größerer Entladungsintensität etwas höher zu sein. Die stärkere Entladung kann dabei sowohl durch Steigerung der Senderleistung, durch Anlegen des statischen Magnetfeldes oder durch leichte Druckerhöhung bedingt sein. Mit Magnetfeld wurden meist mehr als $20 \%$ Protonen gemessen. Ohne Magnetfeld, bei tiefen Drucken und schwachen Senderleistungen betrug der Protonenanteil selten mehr als $\mathbf{1 0 - 1 5 \%}$. Bei etwas höheren

* Anm. b. d. Korr.: Nach neueren Versuchen von $\mathrm{T} h$ on e m a n $\mathrm{n}^{\mathbf{1 2}}$ kann der Anteil an Atomionen dadurch wesentlich gesteigert werden, daß man Metallflächen im Entladungsraum nach Möglichkeit vermeidet oder durch Glas abschirmt.
Drucken wurden gelegentlich über $40 \%$ Protonen beobachtet.*

Außer Molekülionen der Masse 2 und Protonen treten auch bemerkenswert viele Molekülionen der Masse 3 auf. In der der Abb. 7 zugrunde liegenden Messung ist der $\mathrm{H}_{3}^{+}$-Anteil besonders groß. Außerdem wurde die Masse 1 aus der Dissoziation des $\mathrm{H}_{2}$-Moleküls einwandfrei beobachtet (etwa $3-5 \%$ der Häufigkeit der H-Ionen).

Die trotz mäßiger Trennschärfe der Anordnung erzielte gute Trennung der einzelnen Ionengruppen weist darauf hin, daß die Ionen mit praktisch der gleichen Energie aus der Ionenquelle herauskommen.

10. Verhalten der Ionenquelle i m D a u r betrieb, Gasverbrauch

Der Gasverbrauch der Ionenquelle ist im allgemeinen geringer als der eines Kanalstrahlrohrs. Er hängt natürlich vom Durchmesser des Kanals ab. Mittlere Ionenströme sind bei einem Gasverbrauch von $6-10 \mathrm{~cm}^{3}$ Torr/sec erzielt worden. Wenn das Entladungsrohr einige Stunden in Betrieb war oder einen Tag unter Vakuum gestanden hatte, traten kaum mehr Schwankungen der Stromstärke auf. Sorgte man dafür, daß die Anodenplatte gut zentrisch angebracht war, so daß die aus der Entladung zur Anode wandernden Elektronen alle auf die Anodenplatte auftrafen, so traten bei mäßiger Belastung auch bei längerem Betrieb der Röhre keine Schwierigkeiten auf.

12 P. C. Th on emann u. Mitarbb., Proc. Phys. Soc. 61, 483 [1948]. 